\title{
Management of cardiovascular conditions in a cohort of patients with HIV: experience from a joint HIV/cardiology clinic
}

Authors: Sudheer Koganti, ${ }^{\mathrm{A}}$ Sabine Kinloch-de Loes, ${ }^{\mathrm{B}}$ Samantha Hutchinson, ${ }^{\mathrm{C}}$ Margaret Johnson ${ }^{\mathrm{D}}$ and Roby D Rakhit ${ }^{\mathrm{E}}$

The aim of this study was to assess cardiovascular diagnoses and management in a cohort of patients diagnosed with HIV, and the performance of a joint HIV/Cardiology Clinic in a tertiary hospital setting. A retrospective analysis was performed on all patients referred to a joint HIV/Cardiology Clinic at our hospital. Data on 120 patients were collected. In this predominantly male population (male 101 and female 19) coronary artery disease (CAD) was the most common diagnosis $(34 \%, n=41)$. Other diseases included hypertension $(12.5 \%, n=15)$, cardiomyopathy $(12.5 \%, n=15)$ and arrhythmia $(6 \%, n=8)$. The majority of remaining cases included non-cardiac chest pain and palpitations. In addition to usual primary and secondary preventive measures for CAD, complex procedures, such as percutaneous coronary intervention, cardiac resynchronisation therapy for left ventricular systolic dysfunction and radiofrequency ablation for arrhythmias, were carried out. Overall cardiovascular mortality among the group was $2.5 \%$ $(n=3)$ over 4 years. The results indicate the efficacy of a specialist joint HIV/Cardiology Clinic in diagnosing and managing various cardiac conditions in a complex cohort of patients with HIV.

KEYWORDS: HIV, cardiovascular disease, specialist clinic

\section{Introduction}

According to a Public Health England report, in 2012 there were nearly 100,000 HIV cases in the UK. ${ }^{1}$ In the same year, 6,000 new cases were diagnosed, with 490 reported deaths. Advancing

Authors: Aclinical research fellow in cardiology, Department of Cardiology, Royal Free Hospital, London, UK, and UCL Institute of Cardiovascular Science, London, UK; ${ }^{B}$ associate specialist in HIV Medicine, Department of HIV and Infectious Diseases, Royal Free Hospital, London, UK, and senior lecturer, UCL, London, UK; ${ }^{C}$ advanced practitioner HIV medicine, Department of HIV and Infectious Diseases, Royal Free Hospital, London, UK; Dprofessor in HIV medicine, Department of HIV and Infectious Diseases, Royal Free Hospital, London, UK; Econsultant cardiologist, Department of Cardiology, Royal Free Hospital, London, UK, and honorary senior lecturer UCL Institute of Cardiovascular Science, London, UK age, immunosenescence ${ }^{2}$ and prolonged life expectancy in this group due to antiretroviral therapy (ART) will lead to a higher incidence of cardiovascular diseases including coronary artery disease (CAD). ${ }^{3}$ Moreover, inflammation in this cohort of population, as evidenced by ongoing T-cell and monocyte activation, is implemented in the development of non-calcific coronary plaques. ${ }^{4}$ Other diverse cardiovascular manifestations, such as cardiomyopathy, conduction system and metabolic problems, are also increasingly being recognised., 5 Some of the issues involved with effective management of this complex group of patients include: drug interactions, ${ }^{7}$ drug-related side effects ${ }^{8}$ and underestimation of risk of cardiovascular disease by traditional risk scoring systems. ${ }^{9}$ Thus, for optimal management of cardiovascular problems in this group, a multidisciplinary team approach involving cardiologists and HIV specialists is essential. We report our experience of a joint specialist HIV/ Cardiology Clinic and review various cardiovascular diagnoses in this cohort.

\section{Methods}

The clinic

The Royal Free Centre for HIV and the Department of Cardiology run a joint HIV/Cardiology Clinic that was set up in August 2010. The clinic is run by a consultant cardiologist together with an HIV physician in attendance, and supported by a dedicated HIV pharmacist.

\section{Data collection}

Pertinent data over the last 4 years were collected from the electronic records and cardiology reporting systems. Previous letters and procedure reports were analysed to ascertain the accuracy of the data. Details relevant to deaths were obtained from cause of death (CoDe) data maintained by the HIV centre.

\section{Results}

The Royal Free Hospital is a tertiary level hospital in North London looking after 3,245 HIV patients currently. The joint clinic runs once monthly and has received 316 patient visits to date (120 new and 196 follow up). Patients are both internal referrals from physicians working within the HIV centre 
Table 1. Total number of HIV patients and the number referred to joint clinic.

$\begin{array}{lll}\text { Year } & \text { HIV patients, } \mathbf{n} & \begin{array}{l}\text { Patients under the } \\ \text { care of joint clinic, } \mathbf{n}\end{array} \\ 2010 \text { (Aug onwards) } & 2,883 & 13 \\ 2011 & 2,984 & 48 \\ 2012 & 3,086 & 75 \\ 2013 & 3,164 & 105 \\ 2014 \text { (till July) } & 3,245 & 120\end{array}$

and tertiary referrals from outside the centre. Table 1 shows the number of HIV patients looked after every year and the number of referrals to joint clinic year-on-year with a suspected cardiovascular problem. Of the 120 new patients, $101(84 \%)$ were male. The mean age was 52.8 years (standard deviation (SD) 9.1). Fig 1 shows the broad diagnostic groups. Individual diagnostic categories are discussed in detail.

\section{Coronary artery disease}

CAD was the most frequent diagnosis in this cohort. In total, $41(34 \%)$ patients were either diagnosed or referred with suspected CAD. Details of CAD presentation, risk-factor profile and treatments carried out are shown in Table 2. Almost 70\% $(n=24)$ of the patients presented with myocardial infarction (MI) as the initial diagnosis of CAD. These patients presented with $\mathrm{MI}$ at a relatively young age (mean age 48.3 years \pm SD 7.5 ) and were treated with ART for a long period (mean duration $105.7 \pm$ SD 69.7 months). Nearly $80 \%(n=33)$ of the patients were dyslipidaemic and $60 \%(n=25)$ were smokers. Only 2 patients were ART naïve prior to presentation. Nucleoside reverse transcriptase inhibitors (NRTI), either alone or in combination, were the frequently used group of drugs (Table 2). However, only 2/24 patients on NRTI or NRTI combination were on abacavir. The majority of patients were treated with percutaneous coronary intervention, with drug eluting stent being the preferred choice.

\section{Cardiomyopathy}

$15(12.5 \%)$ patients were diagnosed with cardiomyopathy following referral with heart failure symptoms and

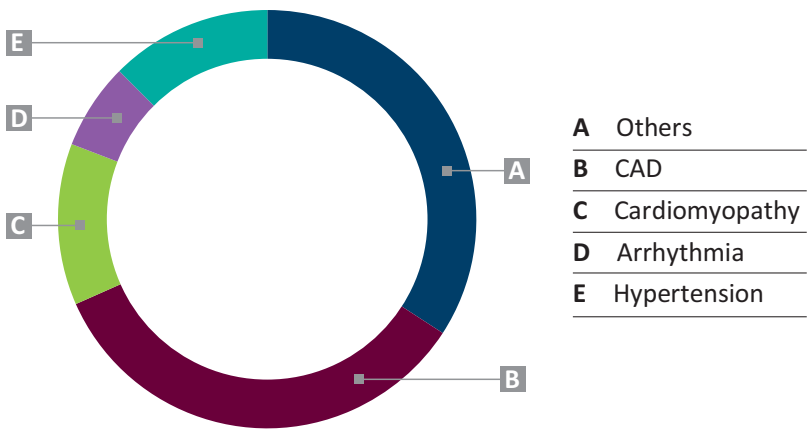

Fig 1. Broad diagnostic groups. $C A D=$ coronary artery disease

\section{Table 2. CAD presentations and treatment.}

$\begin{array}{ll}\text { Characteristic } & \mathbf{n}(\%) \\ \text { CAD } & 34(41) \\ \text { STEMI } & 11 \text { (all und } \\ \text { NSTEMI } & 13 \\ \text { SA } & 11 \\ \text { Asymptomatic CAD } & 6 \\ \text { Current mean age, years } & 51.7 \text { (SD } \\ \text { Mean age at HIV diagnosis, years } & 37.3 \text { (SD } \\ \text { Mean age at CAD diagnosis, years } & 48.3 \text { (SD } \\ \text { Mean duration of antiretroviral therapy, } & 105.7 \text { (SD } 69 \\ \text { months } & \\ \text { Risk factor profile } & \\ \text { Smoker } & 25(61.0) \\ \text { Hypertension } & 21(50.0) \\ \text { Diabetes } & 12(29.0) \\ \text { Dyslipidaemia } & 33(80.0) \\ \text { Family history } & 15(36.0) \\ \text { CKD } & 3(8.8) \\ \text { Hepatitis B/C infection } & 8(23.5) \\ \text { Illicit drug use, including cocaine } & 7(20.5)\end{array}$

Drug history at the time of diagnosis of CAD

$\begin{array}{ll}\text { Antiretroviral drugs } & 32(94) \\ \text { Antihypertensive drugs } & 19(56) \\ \text { Antidiabetic drugs } & 12(35) \\ \text { Lipid lowering drugs } & 21(62)\end{array}$

Frequently used antiretroviral drug groups

$\begin{array}{ll}\text { NRTI alone } & 4(12) \\ \text { NRTI + PI } & 14(41) \\ \text { NRTI + NNRTI } & 4(12) \\ \text { PI + raltegravir } & 4(12) \\ & \\ \text { PCI } & 27(66) \\ \text { OMT } & 12(31) \\ \text { CABG } & 2(3)\end{array}$

$\mathrm{CABG}=$ coronary artery bypass grafting; $\mathrm{CAD}=$ coronary artery disease; $\mathrm{CKD}=$ chronic kidney disease; NNRTI = non-nucleoside reverse transcriptase inhibitors; NRTI = nucleoside reverse transcriptase inhibitors; NSTEMI = non-ST segment elevation myocardial infarction; OMT = optimal medical therapy; $\mathrm{PPCI}=$ primary percutaneous coronary intervention; $\mathrm{PI}=$ protease inhibitors; $\mathrm{SA}=$ stable angina; $\mathrm{SD}=$ standard deviation; STEMI = ST segment elevation myocardial infarction.

abnormal two-dimensional (2D) echocardiography. Dilated cardiomyopathy (DCM) was diagnosed in 9 cases; 7 were based on echocardiography and 2 on the basis of cardiac MRI (CMR). There were 2 cases of ischaemic cardiomyopathy. One case of ischaemic cardiomyopathy was also found to have left ventricular mural thrombus picked up on CMR and appropriately managed with warfarin. In 2 cases, hypertrophic cardiomyopathy was suspected on 2D echocardiography, confirmed on CMR, and appropriately risk stratified. There was one case each of post-partum and chemotherapy-induced cardiomyopathy. All the patients with left ventricular (LV) 
dysfunction were appropriately managed in line with current guidelines, including angiotensin-converting-enzyme inhibitors, beta-blockers and aldosterone antagonists. In addition, 2 patients with DCM underwent cardiac resynchronisation therapy; one with defibrillator and one with just pacing.

\section{Hypertension}

Hypertension was the primary diagnosis in 15 (12.5\%) patients. This number is much higher if the patients with CAD with concomitant hypertension as a risk factor are included. In the majority of the cases no reversible cause was found and thus labelled as essential hypertension. In 1 case, hypertension was secondary to renal artery stenosis. Ambulatory blood pressure monitoring was used in 7 patients to aid in the management.

\section{Arrhythmia}

Cardiac arrhythmia was an important diagnosis seen in this cohort. Almost all were secondary to supraventricular tachyarrhythmia. Details of all 8 cases and treatment are outlined in Table 3. There were no cases of malignant arrhythmia or sudden arrhythmic cardiac death.

\section{Others}

A variety of both common and rare cardiovascular diagnoses totalling $41(34 \%)$ patients were clustered in this group. These are outlined in Table 4

All patients with non-cardiac chest pain were discharged after appropriate investigations with myocardial perfusion scintigraphy, stress echocardiography or computerised tomography coronary angiography, in line with the hospital protocol. 14 patients were referred with palpitations as the main complaint; however history was not suggestive of any arrhythmia. These were reassured and discharged after negative yield on 24-hour Holter monitoring. 1 case of large pericardial cyst of non-specific aetiology required surgical removal in view of pain. 1 case of severe staphylococcal endocarditis required mitral valve replacement. 2 cases of massive pulmonary embolism were jointly managed with cardiologists monitoring for signs of right heart failure. 1 case of amyloid was referred to the national amyloid centre for further management. 2 cases of stroke/transient ischaemic attack with no specific

Table 3. Various rhythm problems and treatment details.

\begin{tabular}{llll} 
Arrhythmia & $\mathbf{n}(\%)$ & Treatment & Success \\
Atrial fibrillation & $3(2.5)$ & $\begin{array}{l}2 \times \text { ablation } \\
1 \times \text { cardioversion }\end{array}$ & Yes \\
Atrial flutter & $2(1.7)$ & $2 \times$ ablation & No \\
AVNRT & $1(0.8)$ & $1 \times$ ablation & Yes \\
PAF & $1(0.8)$ & $\begin{array}{l}\text { Medical therapy } \\
\text { Alcohol abstinence }\end{array}$ & Yes \\
Accessory pathway & $1(0.8)$ & $1 \times$ ablation & Yes \\
\hline
\end{tabular}

AVNRT = atrioventricular nodal re-entrant tachycardia; PAF = paroxysmal atrial fibrillation.

\section{Table 4. Other diagnoses.}

\begin{tabular}{ll} 
Diagnosis & $\mathbf{n}$ \\
Non cardiac chest pain & 14 \\
Palpitations & 14 (normal 24-hour \\
& Holter in all cases) \\
Ventricular septal defect & 2 \\
PE & 2 \\
Stoke/TIA & 2 \\
Cardiac amyloid & 1 \\
Pericardial cyst & 1 (required surgical \\
& removal) \\
Spontaneously resolved pericardial effusion & 1 \\
Aortic stenosis requiring AVR & 1 \\
Abnormal ECG & 1 \\
Infective endocarditis & 1 (required mitral \\
& valve replacement) \\
Syncope & 1 \\
\hline AVR=aortic valve replacement; ECG = electrocardiogram; PE = pulmonary \\
embolism; TIA = transient ischaemic attack.
\end{tabular}

aetiology underwent bubble echocardiography and transoesophageal echo to ensure no cause for paradoxical embolus. 2 cases of ventricular septal defect were monitored, as they were haemodynamically not significant. One case of what was thought to be abnormal electrocardiogram (ECG) was discharged as it turned out to be partial right bundle branch block on cardiology review.

\section{Outcomes}

7 cases of CAD represented with worsening angina or nonST segment elevation myocardial infarction (NSTEMI). 2 cases of NSTEMI underwent further percutaneous coronary intervention (PCI). 2 cases of worsening angina had their medical therapy optimised as the stenosis on angiography was functionally non-significant on pressure wire testing. 1 case had aggressive atherosclerosis and was referred for coronary artery bypass surgery (CABG). A further 2 cases had medical therapy optimised, as there was no progression of disease.

Causes of death were ascertained from the HIV CoDe database. In total there were 5 deaths across the group, of which 3 were cardiovascular. This equates to $2.5 \%$ in this group and $4.3 \%$ of all HIV deaths over the last 4 years. 2 deaths were out-of-hospital: found collapsed at home. The in-hospital death was related to worsening heart failure. Both the out-of-hospital sudden deaths were discussed with the coroner. In 1 case, cause was ascertained due to MI following autopsy and the other case was labelled as death due to hypertensive heart disease with no autopsy.

In the same period, the total number of deaths in the entire HIV cohort under follow up at the Royal Free Hospital was 82 . Of the 82 deaths, 25 were secondary to malignancy, 19 were related to infections, 6 were noted to be due to underlying metabolic problems, and 7 deaths were due to cardiovascular 
and neurological diseases. Further, 10 were attributed to suicide; 2 cases were secondary to road traffic accidents; and cause was not ascertained to 6 cases.

\section{Discussion}

ART has improved the prognosis of HIV-infected patients, not by curing but by converting it into a chronic disease. ${ }^{10}$ This has led to an increased incidence of CAD, partly due to the adverse effects of ART. ${ }^{11}$ The other factor is increasing life expectancy due to ART and consequent overexposure of this group to traditional risk factors of CAD. ${ }^{12}$ As shown in the results section and Table 2, the mean age at the time of diagnosis of CAD was 48.3 years in this cohort. In comparison, the mean age of patients undergoing PCI in England and Wales was 64.9 years as per the British Cardiovascular Interventional Society audit report. ${ }^{13}$ Increased awareness has also lead to identification of a wide range of cardiovascular disease in this group necessitating early specialist input. Atrial arrhythmias, such as atrial fibrillation (AF) and atrial flutter, accounted for $2.6 \%$ of cases in a larger cohort of over 30,000 patients. ${ }^{14}$ In our group, $4.2 \%$ were diagnosed with AF and atrial flutter over 4 years. Low CD4 cell count and high viral load, assessed by multiple timeupdated measures, were shown to be independently associated with development of AF. ${ }^{14}$ However causality is difficult to prove, as other factors such as $\mathrm{CAD}$ and increasing age are noted to be associated with AF. ${ }^{14}$ Two important aspects in the management of atrial arrhythmias are rhythm/rate control and prevention of thromboembolic events. ${ }^{15}$ The drugs used in rate/rhythm control, such as beta-blockers, amiodarone, diltiazem and digoxin, interact with ART, either by inhibiting or inducing the CYP3A4 system. ${ }^{16}$ Anticoagulants, such as warfarin and a new group of anticoagulants that act by direct factor $\mathrm{X}$ inhibition, are used to minimise thromboembolic risk in patients with atrial arrhythmias. Warfarin has the potential to interact with various antiretroviral drugs by induction of CYP3A4 system. ${ }^{5}$ However, the experience with newer anticoagulants such as dabigatran, ${ }^{17}$ rivaroxaban ${ }^{18}$ and apixaban ${ }^{19}$ is limited, and potential interactions need to be watched out for. Incidence of DCM decreased with introduction of ART. ${ }^{20}$ One Italian study diagnosed DCM in $8 \%$ of the patients from a cohort of 952 asymptomatic individuals based on 2D echocardiography. ${ }^{21}$ In our group, $7.5 \%$ patients were diagnosed with DCM. During the initial years we also diagnosed DCM based on echocardiography; however in more recent years this practice has changed to using CMR in view of the diagnostic accuracy and the ability to differentiate between various cardiomyopathies. ${ }^{22,23} \mathrm{CMR}$ would also help in accurate assessment of LV ejection fraction (LVEF) in those with cardiomyopathy, thus guiding the clinician in management decisions. Accurate assessment of LVEF would also have implications on complex device therapy. The National Institute for Health and Care Excellence recommends cardiac resynchronisation therapy (CRT) in patients with severe LV dysfunction symptomatic on optimal medical therapy and certain ECG criteria. ${ }^{24}$ Approximately $6.5 \%$ of AIDS-related mortality is attributable to cardiovascular disease. ${ }^{25} \mathrm{In}$ our cohort it was $4.3 \%$, reflecting high standards of care.

The Royal Free Hospital in North London provides tertiary level care to HIV and cardiology patients. There are currently over 3,200 HIV patients under the care of the HIV centre. Per year, 80-100 new patients are referred from within and outside of London (Table 1). A joint HIV/Cardiology Clinic was set up in August 2010 to cater to the needs of this complex group of patients. The Joint HIV/Cardiology clinic has helped to tap into existing resources and also streamline the process of referral to other hospitals for procedures such as electrophysiology study/ ablation and cardiothoracic surgery. Cases requiring complex interventions, such as multivessel PCI, CABG and CRT device implantation, were facilitated quicker following discussion in respective multidisciplinary team meetings. The polypharmacy used for the treatment and prevention of cardiovascular disease has the potential to interact with ART with the risk of causing toxic effects. ${ }^{5}$ Working closely with a specialist HIV pharmacist allows this risk of drug-drug interaction to be mitigated. The cardiovascular manifestations are wide and varied requiring complex management plans. We have shown from data obtained from the clinic that such an approach is highly effective in managing this unique patient group. This justifies the concept of a joint care model, whereby cardiovascular and HIV specialists work side-by-side to ensure the most appropriate management plan is achieved.

\section{Limitations}

This analysis is limited by a small sample size with no external validation. However, to the best of our knowledge such an experience has not been published before.

\section{References}

1 Public Health England. HIV in the United Kingdom: 2013 report. London: PHE, 2013. Available online at www.gov.uk/government/ uploads/system/uploads/attachment_data/file/326601/HIV_ annual_report_2013.pdf [Accessed 4 August 2015].

2 Tsoukas C. Immunosenescence and aging in HIV. Curr Opin HIV AIDS 2014;9:398-404.

3 Effros RB, Fletcher CV, Gebo K et al. Aging and infectious diseases: workshop on HIV infection and aging: what is known and future research directions. Clin Infect Dis 2008;47:542-53.

4 Grinspoon SK. Cardiovascular disease in HIV: traditional and nontraditional risk factors. Top Antivir Med 2014;22:676-9.

5 Escarcega RO, Franco JJ, Mani BC et al. Cardiovascular disease in patients with chronic human immunodeficiency virus infection. Int J Cardiol 2014;175:1-7.

6 Behrens GM, Meyer-Olson D, Stoll M, Schmidt RE. Clinical impact of HIV-related lipodystrophy and metabolic abnormalities on cardiovascular disease. Aids 2003;17 Suppl 1:S149-54.

7 Aberg JA. Management of dyslipidemia and other cardiovascular risk factors in HIV-infected patients: case-based review. Top HIV Med 2006;14:134-9.

8 Barbaro G, Barbarini G. Highly active antiretroviral therapyassociated metabolic syndrome and cardiovascular risk. Chemotherapy 2006;52:161-5.

9 d'Ettorre G, Francone M, Mancone M et al. Significant coronary stenosis detected by coronary computed angiography in asymptomatic HIV infected subjects. J Infect 2012;64:82-8.

10 Mavroudis CA, Majumder B, Loizides S et al. Coronary artery disease and HIV; getting to the HAART of the matter. Int J Cardiol 2013;167:1147-53.

11 Henry K, Melroe H, Huebsch J et al. Severe premature coronary artery disease with protease inhibitors. Lancet 1998;351:1328.

12 Boccara F, Lang S, Meuleman C et al. HIV and coronary heart disease: time for a better understanding. J Am Coll Cardiol 2013;61:511-23. 
13 British Cardiovascular Intervention Society. National Audit of Percutaneous Coronary Interventional Public Report: January 2012 December 2012. London: NICOR, 2014. Available online at www. hqip.org.uk/assets/NCAPOP-Library/NCAPOP-2013-14/ PCI-Audit-Report-2012.pdf [Accessed 4 August 2015].

14 Hsu JC, Li Y, Marcus GM et al. Atrial fibrillation and atrial flutter in human immunodeficiency virus-infected persons: incidence, risk factors, and association with markers of HIV disease severity. J Am Coll Cardiol 2013;61:2288-95.

15 Camm AJ, Lip GY, De Caterina R et al. 2012 focused update of the ESC guidelines for the management of atrial fibrillation. Eur Heart J 2012;33:2719-47.

16 hiv-druginteractions.org. Drug interaction charts. Available online at www.hiv-druginteractions.org/InteractionCharts.aspx [Accessed 4 August 2015].

17 Hijazi Z, Hohnloser SH, Oldgren J et al. Efficacy and safety of dabigatran compared with warfarin in relation to baseline renal function in patients with atrial fibrillation: a RE-LY (Randomized Evaluation of Long-term Anticoagulation Therapy) trial analysis. Circulation 2014;129:961-70.

18 Patel MR, Mahaffey KW, Garg J et al. Rivaroxaban versus warfarin in nonvalvular atrial fibrillation. N Engl J Med 2011;365:883-91.

19 Granger CB, Alexander JH, McMurray JJ et al. Apixaban versus warfarin in patients with atrial fibrillation. $N$ Engl J Med 2011;365:981-92.
20 Barbaro G. Cardiovascular manifestations of HIV infection. Circulation 2002;106:1420-5.

21 Barbaro G, Di Lorenzo G, Grisorio B, Barbarini G. Incidence of dilated cardiomyopathy and detection of HIV in myocardial cells of HIV-positive patients. N Engl J Med 1998;339:1093-9.

22 White JA, Patel MR. The role of cardiovascular MRI in heart failure and the cardiomyopathies. Cardiol Clin 2007;25:71-95.

23 Satoh H, Sano M, Suwa K et al. Distribution of late gadolinium enhancement in various types of cardiomyopathies: Significance in differential diagnosis, clinical features and prognosis. World $J$ Cardiol 2014;6:585-601.

24 National Institute for Health and Care Excellence. Implantable cardioverter defibrillators and cardiac resynchronisation therapy for arrhythmias and heart failure (review of TA95 and TA120). London: NICE, 2014. Available online at www.nice.org.uk/guidance/TA314/ chapter/1-guidance [Accessed 4 August 2015].

25 Antiretroviral Therapy Cohort Collaboration. Causes of death in HIV-1-infected patients treated with antiretroviral therapy, 19962006: collaborative analysis of $13 \mathrm{HIV}$ cohort studies. Clin Infect Dis 2010;50:1387-96.

Address for correspondence: Dr R Rakhit, Department of Cardiology, Royal Free Hospital, London NW3 2QG, UK. Email: roby.rakhit@nhs.net

\section{Royal College of Physicians Museum and Medicinal Garden}

Visit the Royal College of Physicians (RCP) and discover 500 years of medical history in an acclaimed architectural setting.

The RCP is the oldest medical college in England, and since its foundation in 1518 it has built up magnificent collections of books, manuscripts, portraits, silver, and medical artefacts.

Our celebrated Grade I listed headquarters in Regent's Park is the creation of British architect Sir Denys Lasdun and our garden contains over 1,300 plants, each used as medicine around the world over the past 3,000 years.

Guided tours

We offer guided tours of the building and garden, and welcome bookings for groups of six or more. Refreshments can be booked and tours tailored for special interest groups. Guided tour charges are $€ 5$ per person (refreshments extra).

\section{Royal College of Physicians, 11 St Andrews Place, Regent's Park, London NW1 4LE. Open: Monday- Friday, $9 \mathrm{am}-5 \mathrm{pm}$, free entry \\ Library, Archive and Museum Services \\ Tel: +44 (0)203075 1543 \\ Email: history@rcplondon.ac.uk}

\section{Free} entry

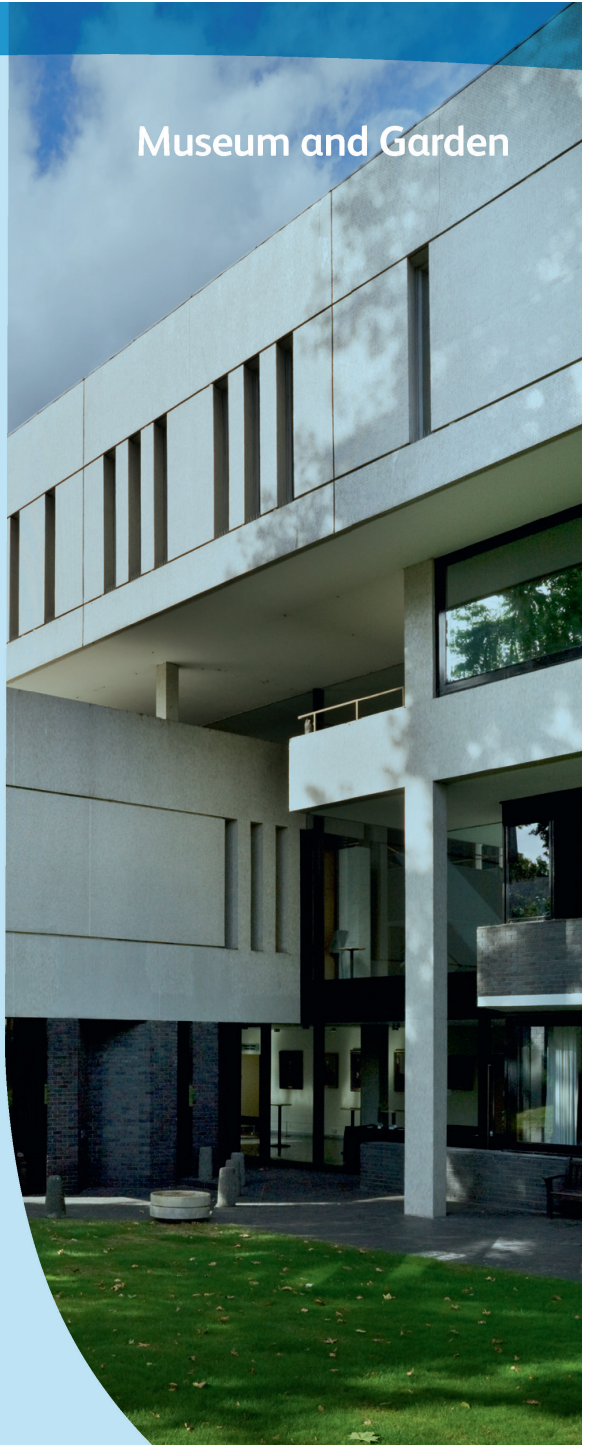

